\title{
Isolated septic arthritis of hip joint: a rare presentation of melioidosis. A case report
}

(a) CrossMark

\author{
N. P. Weerasinghe ${ }^{1 *}$, H. M. M. Herath ${ }^{2,3}$ and T. M. U. Liyanage ${ }^{3}$
}

\begin{abstract}
Background: Despite, Sri Lanka lies in the melioidosis endemic belt between $5^{\circ} \mathrm{N}$ and $10^{\circ} \mathrm{N}$ surrounded by countries known to have endemic melioidosis for many years, comparatively fewer cases of melioidosis infection have been reported in Sri Lanka. Melioidosis has a wide spectrum of clinical presentation, ranging from severe pneumonia to abscess formation in various organs. Isolated septic arthritis, which is a rare but well-recognized manifestation of melioidosis, could be the sole presenting problem in some patients with melioidosis.

Case presentation: We report a middle aged diabetic female who has been on azathioprine for autoimmune hepatitis, presenting with pain and swelling of left hip joint. Investigations confirmed the clinical suspicion of septic arthritis, but all relevant microbiological investigations failed to isolate a causative organism. Due to the history of diabetes, possible immunosuppression with azathioprine, and failure to recognise the possible causative organism by initial investigations prompted us to investigate for melioidosis. Diagnosis of melioidosis was made by presence high titre of antibodies to melioidin antigen, and rapid response to appropriate treatment. The patient was treated with intravenous imipenem 1000 mg 6 hourly and oral cotrimoxazole (1920 mg 12 hourly) for 4 weeks followed by eradication therapy with cotrimoxazole and doxycycline.

Conclusion: Given that melioidosis-induced septic arthritis share common features with septic arthritis due to other common pyogenic bacteria, differentiation of these two conditions is extremely difficult. Therefore, melioidosis needs to be considered as a possibility, when a patient with risk factors for melioidosis such as diabetes or immunosuppression presents with isolated septic arthritis. This case report has been presented to raise the awareness of an unusual presentation of melioidosis; isolated septic arthritis.
\end{abstract}

Keywords: Melioidosis, Isolated septic arthritis, Immune suppression, Sri Lanka

\section{Background}

Melioidosis is a pyogenic infection with high mortality and is caused by the facultative intracellular gram-negative bacterium; Burkholderia pseudomallei $[1,2]$. It is endemic in tropical and subtropical zones of South East Asia and Northern Australia [2]. Despite, Sri Lanka lies in the melioidosis-endemic belt between $5^{\circ} \mathrm{N}$ and $10^{\circ} \mathrm{N}$ surrounded by countries known to have endemic melioidosis for many years, comparatively fewer cases of melioidosis infection have been reported in Sri Lanka [3]. Commonly identified risk factors for this infection are diabetes

\footnotetext{
*Correspondence: herathtpw@gmail.com

1 Department of Microbiology, Faculty of Medicine, University of Ruhuna, P.O. Box 70, Galle, Sri Lanka

Full list of author information is available at the end of the article
}

mellitus, heavy alcohol use, malignancy, chronic lung, liver and kidney disease and various other immune suppressive conditions [1]. Burkholderia pseudomallei enters the body through percutaneous inoculation or inhalation [1]. The disease is known as a remarkable imitator of other diseases such as tuberculosis, due to the wide and variable clinical spectrum of its manifestations [2]. As a result, a high index of clinical suspicion is required for the diagnosis. Delay in the diagnosis and failure to start appropriate and effective treatment against melioidosis, can worsen the outcome $[4,5]$.

When compared with other bacterial infections, melioidosis is a less common cause for isolated septic arthritis. Even though, melioidotic bone and joint infections are established entities, they are less commonly reported 
than other manifestations of melioidosis [6, 7]. Knee joint has been identified as the most commonly affected joint in melioidosis, followed by ankle, hip and shoulder joints [6]. Even though, melioidotic bone and joint infections have been reported in Sri Lanka previously [3, 8-10], isolated septic arthritis as the sole manifestation of melioidosis has not been reported in Sri Lanka up to now. Therefore, we report this unusual case of isolated septic arthritis of left hip joint due to melioidosis in a 45-year old female with multiple risk factors.

\section{Case presentation}

A 45-year old Sinhalese woman from Galle district in Southern Province of Sri Lanka presented to our hospital with a 2 week history of intermittent fever and severely painful left hip for 3 days duration. She had a history autoimmune hepatitis for 6 months, for which she had been on azathioprine $50 \mathrm{mg}$ daily, and poorly controlled type 2 diabetes mellitus for 11 years.

Fever was intermittent, initially low grade and later high grade, which abated with sweating, but not associated with chills and rigors. She also had mild sore throat and cough with constitutional symptoms. She was initially managed with oral co-amoxyclav as for possible upper respiratory tract infection by her family doctor. However, her fever continued and around 3 days prior to the admission, she has noticed severe pain in her left hip joint which has caused her to be increasingly bedbound and restricted left hip movement. Apart from these symptoms, she did not have involvement of other joints or haemoptysis. There was no previous history of tuberculosis or close contact with a patient with tuberculosis. She was a field officer, and had no exposure to soil; however, she occasionally had engaged in gardening.

On examination, she was ill looking and had a temperature of $40{ }^{\circ} \mathrm{C}$. Examination revealed severely restricted movements of left hip. The joint was mildly warm to touch but there was no obvious swelling over it. Initial laboratory investigations revealed raised peripheral white cell count of $12.4 \times 10^{9} / \mathrm{L}$ with $82 \%$ polymorphonuclear leukocytes, hemoglobin of $9.1 \mathrm{~g} / \mathrm{dL}$ and a normal platelet count. Both erythrocyte sedimentation rate $(65 \mathrm{~mm} / 1 \mathrm{st} \mathrm{h})$ and C-reactive protein $(86 \mathrm{mg} / \mathrm{dL})$ were elevated. Initial radiograph of left hip joint was normal, but subsequent $\mathrm{x}$-ray which was taken 1 week later showed a narrow joint space and destruction of femoral head (Fig. 1). Ultra sound scan of hip revealed small effusion with echogenic particles. MRI of hip was also suggestive of septic arthritis (Fig. 2).

After taking blood cultures, she was commenced on empirical antibiotic therapy with ceftriaxone IV $1 \mathrm{~g} 12$ hourly and vancomycin IV $1 \mathrm{~g} 12$ hourly. Despite antibiotic therapy, high fever spikes were continued and

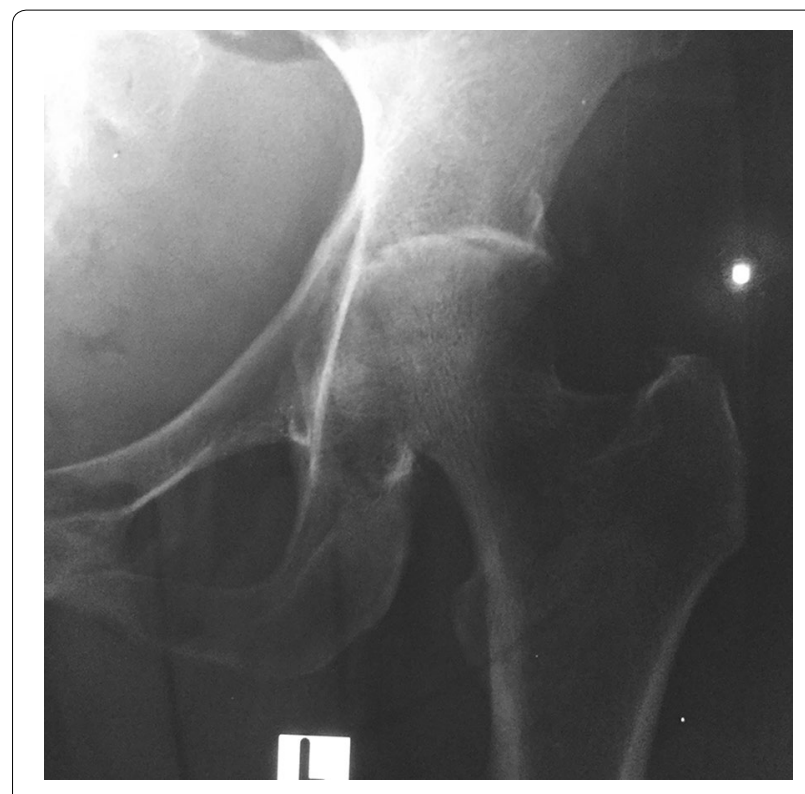

Fig. 1 X-ray of the left hip showing narrow joint space and destruction of femoral head

the clinical condition deteriorated. Repeated blood cultures and urine cultures revealed negative results. Joint fluid aspiration was failed since there was only a minimal amount of fluid. Further investigations including Mantoux test and sputum for acid fast bacilli (AFB) and mycobacterial culture were negative. Transthoracic 2D-echocardiogram did not show vegetations. Chest $x$-ray, ultrasound scan of abdomen and subsequently contrast CT scan of chest and abdomen did not reveal any significant abnormality such as visceral lymphadenopathy or abscesses. Autoimmune panel including ANA, ANCAs and serology for other less common infective causes like HIV, toxoplasmosis, and brucellosis were also negative.

Due to her immunosuppressive state and unresponsiveness to empirical antibiotic regimen, possibility of uncommon infective conditions was considered at this stage. One of the conditions that we considered was melioidosis since it is known to cause isolated septic arthritis in susceptible patients. Since the blood cultures were repeatedly negative, we performed antibodies to $B$. pseudomallei, which came positive with a titre of $1 / 10240$ by Indirect Haemagglutination Assay (IHA). The presumptive diagnosis of melioidosis was made based on clinical and microbiological grounds. Definitive treatment was started with intravenous imipenem $1000 \mathrm{mg} 6$ hourly and oral co-trimoxazole (1920 mg 12 hourly). The patient responded well to the above treatment with fever settling within $72 \mathrm{~h}$ of starting treatment. While in the ward, she had intensive physiotherapy to improve mobilisation. At 


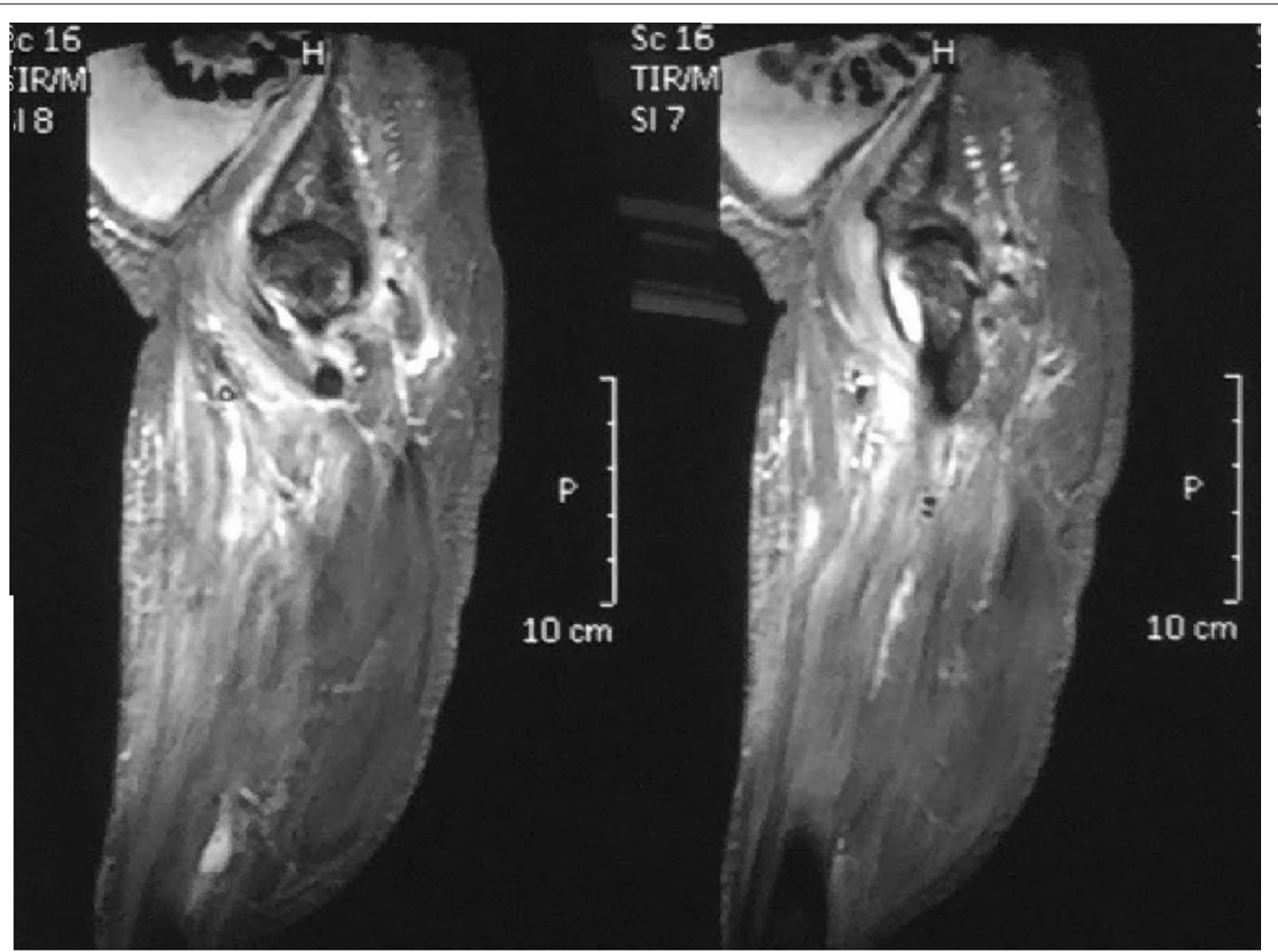

Fig. 2 A coronal enhanced T1-weighted MRI image shows severe erosive bone changes, diffuse joint space narrowing and a small amount of joint effusion with synovial enhancement

the end of 3 weeks, inflammatory markers came down to normal range and she was discharged on oral cotrimoxazole for another 10 weeks with a plan to have weekly reviews with full blood count and CRP.

\section{Discussion and conclusions}

Melioidosis is caused by the gram-negative saprophyte Burkholderia pseudomallei [1] and it is mainly endemic to South East Asia and Northern Australia [2]; however, in recent years, there is increasing number of cases of melioidosis reported in Sri Lanka [3, 8, 11-14]. The spectrum of disease varies from mild self-limiting fever to severe septicemia with abscess formation in several organs [1]. It carries a high mortality unless treated early with appropriate antimicrobials.

Septic arthritis is a rare, but well recognized manifestation of melioidosis [6]. Clinically, it is difficult to differentiate from septic arthritis due to other common pathogens like Staphylococcus aureus or Haemophilus influenzae. Similar to other common pathogens, Burkholderia pseudomallei also affects large joints, such as knee, ankle, elbow, hip and shoulder [7]. However, unlike in our patient, knee joint is reported to be the most commonly involved joint in melioidosis [6]. However, a study conducted by Teparrakkul et al. [7] showed higher incidence of lower extremity joint involvement. In our patient, hip joint was the only affected joint. Even from Northern Australia and Thailand, where melioidosis is highly endemic, there is a paucity of evidence of such isolated involvement of hip joint. As far as we are aware, there have been no reported cases of isolated septic arthritis due to melioidosis, without other organ involvement from Sri Lanka. In one of the case series involving 540 patients, only four patients $(<1 \%)$ had septic arthritis [2]. Interestingly, in these case series conducted in different settings, blood cultures were reported to be positive in all patients with melioidotic septic arthritis $[2,15]$. In contrast, blood cultures of our patient were repeatedly negative, and this could be due to prior antibiotic use. Even though, isolation of B. pseudomallei from the body fluids of patients remains the gold standard in the diagnosis, IHA has been used as the main serologic assay worldwide $[16,17]$. Specificity of IHA is largely dependent on the prevalence of background sero-positivity. As it is high in endemic areas, IHA should be used cautiously in the diagnosis of melioidosis in endemic regions [16]. However, it may have a place in the diagnosis of active infection in non-endemic areas $[17,18]$. Even though, Sri Lanka is now considered as endemic area with increasing number of cases reported in last decade, IHA may 
still be useful in selected patients like ours if the clinical suspicion is high. Diagnosis of melioidotic septic arthritis is made in our patient based on the presence of very high titre of antibody (IHA), good response to specific treatment targeting melioidosis and exclusion of other possible causative organisms.

One of the most important risk factor for melioidotic septic arthritis is diabetes mellitus; however, underlying immunosuppression is also an important risk factor $[6$, 15]. Our patient had both these risk factors. In melioidotic joint infections, the presenting symptom is usually persistent fever rather than shock or respiratory failure, and overall mortality is low among patients with melioidotic joint infections [7]. Our patient too had persistent fever with no evidence of haemodynamic compromise or respiratory distress. Even with significant damage to the hip joint, our patient had a remarkable recovery and was able to walk with minimal assistance at the end of 3 weeks of treatment.

In conclusion, isolated septic arthritis, a rare but a recognized manifestation of melioidosis, could be the presenting problem in some patients with melioidosis. This interesting case highlights the importance of considering "melioidosis" in susceptible patients presenting with isolated septic arthritis, even if the other manifestations of melioidosis (abscess, lung involvement) are not present. Our case report also illustrates the difficulty in isolating B. pseudomallei especially when there is prior antibiotic use. This case further illustrates the fact that the IHA, even though not very specific test particularly in endemic areas, can be used cautiously to diagnose melioidosis.

\section{Abbreviations}

AFB: acid fast bacilli; 2D-echocardiogram: two-dimensional echocardiography; CT: computed tomography; ANA: antinuclear antibody; ANCAs: anti-neutrophil cytoplasmic antibodies; HIV: human immunodeficiency virus.

\section{Authors' contributions \\ HMMH was involved in diagnosis, management and writing the manuscript. TMUL was involved in investigations and writing the manuscript. NPW was involved in the management of the patient and writing the manuscript. All authors read and approved the final manuscript.}

\section{Author details}

${ }^{1}$ Department of Microbiology, Faculty of Medicine, University of Ruhuna, P.O. Box 70, Galle, Sri Lanka. ${ }^{2}$ Department of Medicine, Faculty of Medicine, University of Ruhuna, Galle, Sri Lanka. ${ }^{3}$ University Unit, Teaching Hospital, Karapitiya, Galle, Sri Lanka.

\section{Acknowledgements}

The authors wish to acknowledge the patient profiled in this case report for permitting us to publish this case. The authors would also like to acknowledge all the staff of university medical unit of Teaching Hospital Karapitiya for their assistance in investigating this patient.

\section{Competing interests}

The authors declare that they have no competing interests.

\section{Availability of data and materials}

Data sharing is not applicable to this article, as no datasets were generated or analyzed. This patient's hospital record is not shared as it contains personal identification information.

\section{Consent to publish}

Written informed consent was obtained from the patient to publish of this case report and the accompanying images. A copy of the written consent is available for review by the Editor-in-Chief of this journal.

Ethics approval and consent to participate

Not applicable.

\section{Funding}

This was an unfunded study.

\section{Publisher's Note}

Springer Nature remains neutral with regard to jurisdictional claims in published maps and institutional affiliations.

Received: 19 June 2017 Accepted: 12 January 2018

Published online: 19 January 2018

\section{References}

1. Cheng AC, Currie BJ. Melioidosis: epidemiology, pathophysiology, and management. Clin Microbiol Rev. 2005;18(2):383-416.

2. Currie BJ, Ward L, Cheng AC. The epidemiology and clinical spectrum of melioidosis: 540 cases from the 20 year Darwin prospective study. PLoS Negl Trop Dis. 2010;4(11):e900.

3. Corea EM, Merritt AJ, Ler YH, Thevanesam V, Inglis TJ. Sri Lankan national melioidosis surveillance program uncovers a nationwide distribution of invasive melioidosis. Am J Trop Med Hyg. 2016;94(2):292-8.

4. Raja NS, Ahmed MZ, Singh NN. Melioidosis: an emerging infectious disease. J Postgrad Med. 2005;51(2):140-5.

5. Chaowagul W, White NJ, Dance DA, Wattanagoon Y, Naigowit P, Davis TM, Looareesuwan S, Pitakwatchara N. Melioidosis: a major cause of community-acquired septicemia in northeastern Thailand. J Infect Dis. 1989;159(5):890-9.

6. Kosuwon W, Taimglang T, Sirichativapee W, Jeeravipoolvarn P. Melioidotic septic arthritis and its risk factors. J Bone Jt Surg. 2003;85-a(6):1058-61.

7. Teparrakkul P, Tsai JJ, Chierakul W, Gerstenmaier JF, Wacharaprechasgu T, Piyaphanee W, Limmathurotsakul D, Chaowagul W, Day NP, Peacock SJ. Rheumatological manifestations in patients with melioidosis. Southeast Asian J Trop Med Public Health. 2008:39(4):649-55.

8. Caldera AS, Kumanan T, Corea E. A rare cause of septic arthritis: melioidosis. Trop Dr. 2013;43(4):164-6.

9. Mathurageethan M, Kahathuduwa C, Badanasinghe N, Corea E, Fernando R. Melioidosis associated with chronic osteomyelitis and visceral organ abscesses. Sri Lanka J Surg. 2014;32(2):41-2.

10. Rodrigo KMD, Premaratna R, de Silva HJ, Corea E. Melioidosis as a cause of femoral osteomyelitis and multifocal intramuscular abscess around the hip joint in a farmer: a case report. Sri Lankan J Infect Dis. 2013;3(1):50-4.

11. Fonseka CL, Galappaththi SR, Illagatilaka A, Dasanayake D, Tissera N Acute pulmonary melioidosis presenting with multiple bilateral cavitary lesions in a healthy young adult: an authentic case report from Sri Lanka. BMC Res Notes. 2016;9:360.

12. Wijekoon S, Prasath T, Corea EM, Elwitigala JP. Melioidosis presenting as lymphadenitis: a case report. BMC Res Notes. 2014;7(1):364.

13. Kahandawaarachchi ICI, Premawansa GS, Warnasuriya W, Dassanayake M, Corea E. A case report of co-infection of melioidosis and cutaneous Leishmaniasis. BMC Infect Dis. 2017;17:533.

14. Nandasiri S, Wimalaratna H, Manjula M, Corea E. Transverse myelitis secondary to melioidosis: a case report. BMC Infect Dis. 2012;12:232.

15. Kingsley PV, Leader M, Nagodawithana NS, Tipre M, Sathiakumar N. Melioidosis in Malaysia: a review of case reports. PLoS Negl Trop Dis. 2016:10(12):e0005182. 
16. Limmathurotsakul D, Chantratita N, Teerawattanasook N, Piriyagitpaiboon K, Thanwisai A, Wuthiekanun V, Day NP, Cooper B, Peacock SJ. Enzymelinked immunosorbent assay for the diagnosis of melioidosis: better than we thought. Clin Infect Dis. 2011;52(8):1024-8.

17. Cheng AC, O’Brien M, Freeman K, Lum G, Currie BJ. Indirect hemagglutination assay in patients with melioidosis in northern Australia. Am J Trop Med Hyg. 2006;74(2):330-4.
18. Van Peenen PF, See R, Soysa PE, Irving GS. Seroepidemiological survey of hospital-associated populations in Colombo, Sri Lanka. Southeast Asian J Trop Med Public Health. 1976;1:16-20.

\section{Submit your next manuscript to BioMed Central} and we will help you at every step:

- We accept pre-submission inquiries

- Our selector tool helps you to find the most relevant journal

- We provide round the clock customer support

- Convenient online submission

- Thorough peer review

- Inclusion in PubMed and all major indexing services

- Maximum visibility for your research

Submit your manuscript at

www.biomedcentral.com/submit
() Biomed Central 